\title{
Superficial Acral Fibromyxoma involving the nail's apparatus. Case report and literature review ${ }^{*}$
}

\author{
Ana Márquez García ${ }^{1}$ \\ Manuel Perea Cejudo ${ }^{3}$ \\ Juan José Ríos Martín
}

\author{
Francisco Manuel Ildefonso Mendonça ${ }^{2}$ \\ Francisco M. Camacho Martínez ${ }^{4}$
}

DOI: http://dx.doi.org/10.1590/abd1806-4841.20142673

\begin{abstract}
Superficial Acral Fibromyxoma is a rare tumor of soft tissues. It is a relatively new entity described in 2001 by Fetsch et al. It probably represents a fibrohistiocytic tumor with less than 170 described cases. We bring a new case of SAF on the 5th toe of the right foot, in a 43-year-old woman. After surgical excision with safety margins which included the nail apparatus, it has not recurred (22 months of follow up). We carried out a review of the location of all SAF published up to the present day.
\end{abstract}

Keywords: Nails; Neoplasm recurrence, local; Neoplasms; Recurrence

\section{INTRODUCTION}

Superficial Acral Fibromyxoma (SAF) is a rare tumor of soft tissues with slow growth and acral location. It has a benign behavior, but it may persist or recur if not properly treated.

\section{CASE REPORT}

We present a 43-year-old woman, without any known allergies whose personal history reports beta thalassemia. She referred having had cutaneous changes not associated with any trauma for 8 years, consisting of swelling, partial nail loss and distal ulcerations with occasional bleeding on the $5^{\text {th }}$ toe of the right foot. When the patient wore open shoes it was painless; however, it hurt and bled when she wore closed shoes. Upon examination, the distal end of the $5^{\text {th }}$ toe presented a central ulcer with blood remains and partial onycholysis (Figure 1A).

Antero-posterior and oblique X-rays were requested of both feet, which showed subluxation of the distal phalanges of the $5^{\text {th }}$ toes without signs of bone infiltration and a diagnostic biopsy was performed.

Histological results showed neoplastic dermal proliferation of fusiform cells without any relevant atypia, immersed in a myxoid stroma with collagenized areas and a prominent vascular weave (Figure 1B).
Immunohistochemical studies reported positive results for CD34 and negative for S100, AME and AML (Figure 1C). The proliferation index, valued with Ki67 was low (less than 1\%). These findings led to the diagnosis of Superficial Acral Fibromyxoma.

The subsequent therapeutic approach included complete removal of the tumor as well as the nail in order to avoid recurrence.

Histological examination of the surgical piece was similar to the previously described. The tumor was extirpated with wide margins, including the nail matrix, respecting the distal phalanx (Figure 1D). Resection margins were reported as tumor-free.

After a 22-month follow-up there was no recurrence of the tumor.

\section{DISCUSSION}

Superficial Acral Fibromyxoma (SAF) is a rare tumor of soft tissues, with slow growth and located in the subungual or periungual region of the hands and feet $^{1-7}$ (Table 1). However, the heel, palm and ankle can also be affected. ${ }^{5}$ It affects young adults (mean age 43 years old), with higher frequency in men than in women in a 2:1 proportion. It is a relatively new entity described in 2001 by Fetsch et al. ${ }^{4}$ It probably repre-

Received on 04.04.2013.

Approved by the Advisory Board and accepted for publication on 17.07.2013.

**Work performed at the Hospital Universitario Virgen Macarena - Sevilla, Spain.

Financial Support: none

Conflict of Interests: none

MD - EIR 4th year Dermatology, Hospital Universitario Virgen Macarena - Sevilla, Spain.

MD - EIR 2nd year Dermatology, Hospital Universitario Virgen Macarena - Sevilla, Spain.

MD, Specialist in Dermatology. - Adjunct Physician at Department of Dermatology, Hospital Universitario Virgen Macarena - Sevilla, Spain.

MD, Specialist in Dermatology. PhD in Medicine. - Professor of Dermatology, Hospital Universitario Virgen Macarena - Sevilla, Spain.

MD, Specialist in Pathological Anatomy. - Adjunct Physician of Pathological Anatomy, Hospital Universitario Virgen Macarena - Sevilla, Spain.

C2013 by Anais Brasileiros de Dermatologia 

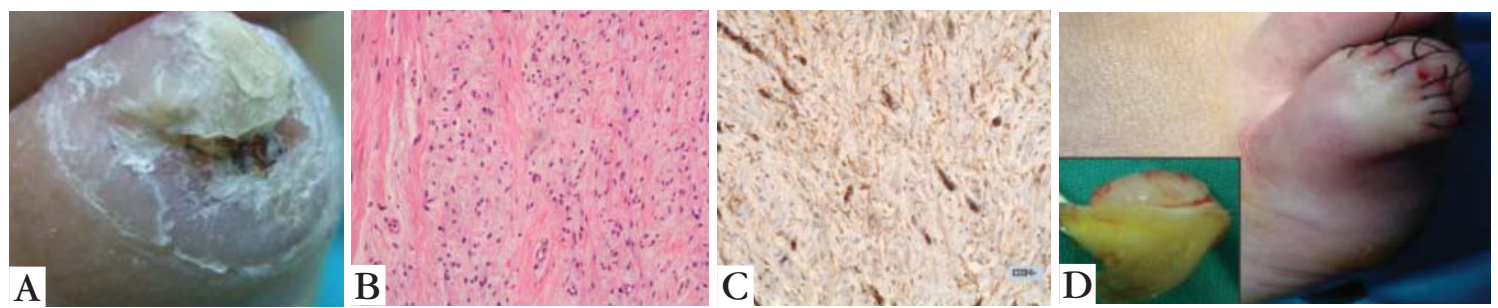

FIGURE 1: A: Loss of the distal end of the 5 th toe of the right foot and partial onycholysis; B: Fusiform cell proliferation in a myxoid stroma (HE, x100); C: Fusiform cells show cytoplasmic positivity to CD34 (PAP, x200); D: Image after excision. The macroscopic part of the tumor along with nail apparatus can be seen

TABLE 1: Summary of all published cases and locations of SAF

\begin{tabular}{|c|c|c|c|c|c|c|c|}
\hline References & $\begin{array}{l}\text { Cases } \\
\text { described }\end{array}$ & Location & $\mathrm{N}^{\circ}$ Cases & References & $\begin{array}{l}\text { Cases } \\
\text { described }\end{array}$ & Location & $\mathrm{N}^{\circ}$ Cases \\
\hline Fetsch JF et al. & 37 & $\begin{array}{l}\text { Toes } \\
\text { Fingers }\end{array}$ & $\begin{array}{l}20 \\
13\end{array}$ & $\begin{array}{l}\text { Tardío JC et al. } \\
\text { Am J Dermatopathol. }\end{array}$ & 4 & $\begin{array}{l}\text { Big toe } \\
\text { Middle finger }\end{array}$ & $\begin{array}{l}1 \\
1\end{array}$ \\
\hline $\begin{array}{l}\text { Hum Pathol. 2001; } \\
\text { 32:704-14. }\end{array}$ & & Palm & 4 & $2008 ; 30: 431-5$ & & $\begin{array}{l}\text { Palm of hand } \\
\text { Thumb of hand }\end{array}$ & $\begin{array}{l}1 \\
1\end{array}$ \\
\hline $\begin{array}{l}\text { Kazakov DV et al. } \\
\text { Dermatology. 2002; } \\
\text { 205:285-8. }\end{array}$ & 2 & Toes & 2 & $\begin{array}{l}\text { Luzar B and Calonje E. } \\
\text { Histopathology. } \\
\text { 2009; 54:375-7. }\end{array}$ & 14 & $\begin{array}{l}\text { Big toe } \\
i\end{array}$ & $\begin{array}{l}8 \\
6\end{array}$ \\
\hline $\begin{array}{l}\text { Meyerle JH et al. } \\
\text { J Am Acad Dermatol. } \\
\text { 2004; 50:134-6. }\end{array}$ & 1 & $\begin{array}{l}\text { Subungueal } \\
\text { index finger }\end{array}$ & 1 & $\begin{array}{l}\text { Pasquinelli G et al. } \\
\text { Ultrastruct Pathol. } \\
\text { 2009; 33:293-301. }\end{array}$ & 1 & Index finger & 1 \\
\hline $\begin{array}{l}\text { André J et al. } \\
\text { Am J Dermatopathol. } \\
\text { 2004; 26:472-4. }\end{array}$ & 1 & Great toe-nail & 1 & $\begin{array}{l}\text { Wang QF et al. Zhonghua } \\
\text { Bing Li Xue Za Zhi. } \\
\text { 2009; 38:682-5. }\end{array}$ & 1 & Middle finger & 1 \\
\hline $\begin{array}{l}\text { Quaba O et al. } \\
\text { Br J Plast Surg. } \\
\text { 2005; 58:561-4. }\end{array}$ & 1 & Ring finger & 1 & $\begin{array}{l}\text { Goo J et al. Ann Dermatol. } \\
\text { 2010; 22:110-3. }\end{array}$ & 1 & $\begin{array}{l}\text { Subungueal } \\
\text { index finger }\end{array}$ & 1 \\
\hline $\begin{array}{l}\text { Abou-Nukta F et al. } \\
\text { J Hand Surg Br. } \\
\text { 2006; 31:619-20. }\end{array}$ & 1 & Nail of the thumb & 1 & $\begin{array}{l}\text { Chattopadhyay M et al. } \\
\text { Clin Exp Dermatol. } \\
\text { 2010;35:807-9. }\end{array}$ & 1 & $\begin{array}{l}\text { Subungueal } \\
\text { index finger }\end{array}$ & 1 \\
\hline $\begin{array}{l}\text { Oteo -Alvaro A et al. } \\
\text { Arch Orthop Trauma } \\
\text { Surg. 2008; 128:271-4. }\end{array}$ & 1 & Toe & 1 & $\begin{array}{l}\text { Cogrel O et al. Ann } \\
\text { Dermatol Venereol. } \\
\text { 2010; 137: 789-93. }\end{array}$ & 3 & $\begin{array}{l}\text { Great toe } \\
\text { Second Toe } \\
\text { Finger }\end{array}$ & $\begin{array}{l}1 \\
1 \\
1\end{array}$ \\
\hline $\begin{array}{l}\text { Misago N et al. } \\
\text { J Eur Acad Dermatol } \\
\text { Venereol. 2008; 22:255-7. }\end{array}$ & 1 & Tip of big toe & 1 & $\begin{array}{l}\text { Fanti PA et al. } \\
\text { G Ital Dermatol Venereol. } \\
\text { 2011;146: 283-7. }\end{array}$ & 12 & $\begin{array}{l}\text { Toes } \\
\text { Fingers }\end{array}$ & $\begin{array}{l}i \\
i\end{array}$ \\
\hline $\begin{array}{l}\text { Varikatt W et al. } \\
\text { Skeletal Radiol. } \\
\text { 2008; 37:499-503. }\end{array}$ & 2 & Tip of index finger & 2 & $\begin{array}{l}\text { Messeguer F et al. } \\
\text { Actas Dermosifiliogr. } \\
\text { 2012; 103:67-9. }\end{array}$ & 1 & Index finger & 1 \\
\hline $\begin{array}{l}\text { Al-Daraji WI et al. } \\
\text { J Cutan Pathol. } \\
\text { 2008; 35:1020-6. }\end{array}$ & 32 & $\begin{array}{l}\text { Toes } \\
\text { Fingers } \\
\text { Heel }\end{array}$ & $\begin{array}{l}15 \\
13 \\
4\end{array}$ & $\begin{array}{l}\text { Ben Brahim E et al. } \\
\text { Tunis Med. 2012;90:340-1. }\end{array}$ & 1 & Toe & 1 \\
\hline $\begin{array}{l}\text { Al-Daraji WI et al. } \\
\text { Dermatol Online J. } \\
\text { 2008; 28:14-27. }\end{array}$ & 2 & $\begin{array}{l}\text { Subungueal big toe } \\
\text { Index finger }\end{array}$ & $\begin{array}{l}1 \\
1\end{array}$ & $\begin{array}{l}\text { Wakabayashi Y et al. } \\
\text { Acta Dermatovenerol } \\
\text { Croat. 2012; 20:263-6. }\end{array}$ & 1 & Great toe & 1 \\
\hline $\begin{array}{l}\text { Prescott RJ et al. } \\
\text { Br J Dermatol. } \\
\text { 2008; 159:1315-21. }\end{array}$ & 41 & $\begin{array}{l}\text { Toes } \\
\text { Fingers } \\
\text { Palm }\end{array}$ & $\begin{array}{l}29 \\
11 \\
1\end{array}$ & $\begin{array}{l}\text { Wei C et al. Eplasty. } \\
\text { 2013; 13: ic13. }\end{array}$ & 1 & Thumb & 1 \\
\hline
\end{tabular}


Chart 1: Differential Diagnosis of Superficial Acral Fibromyxoma

\begin{tabular}{|lll}
\hline CD34+ Neoplasias & CD34- Neoplasias & Other lesions \\
\hline Dermatofibrosarcoma protuberans & Giant cell tumor of tendon sheath & Fibroma of tendon sheath \\
Superficial angiomyxoma & Glomus tumor & Onychocryptosis \\
Myxoid neurofibroma & Sclerosing perineuroma & Cutaneous muxoma \\
Sclerosing fibroma & Benign fibrous histiocytoma & \\
Acral myxoinflammatory & Acral fibrokeratoma & \\
Spindle cell lipoma & & \\
\hline
\end{tabular}

sents a fibrohistiocytic tumor with less than 170 described cases (SAF series and isolated case reports). ${ }^{2}$

Pain is not usually mentioned. Ungual involvement may be present. Only one case has been associated with previous trauma. X-rays rarely show bone

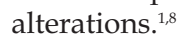

Histologically, it is a well delimited, non-encapsulated dermal tumor that may extend towards the hypodermis. It is composed of a proliferation of cells from a fibroblastic line usually accompanied by many mast cells. The presence of a myxoid stroma with a rich vascular weave is very noticeable. Epidermis hyperplasia with hyperkeratosis is also frequent. CD34 positivity is characteristic but CD10, CD99, EMA, and nestin immunoreactivity are also common. Negative results for neural and muscular differentiation markers (S-100, HMB-45, SMA, desmin, actin), cytokeratin and apolipoprotein D are expected. ${ }^{1-3,9,10}$

Although it is an infrequent event, it must be included in differential diagnosis of tumors present on the fingers and toes. ${ }^{1-7}$ Differential diagnosis considerations are summarized in chart 1 .

SAF has a benign behavior but may persist or recur if not properly treated. ${ }^{7,8}$ Thus complete removal and follow-up is recommended. Up to this date, malignization has not been described.

In conclusion, this is the description of a rare case of Superficial Acral Fibromyxoma on the nail apparatus of a 43-year-old woman. There are less than 170 published cases. It is a benign tumor with slow growth and, although rare, it should be considered in differential diagnosis of acral lesions. Surgery is curative but requires adequate margins due to the high risk of recurrence. Malignization has never been described.

\section{REFERENCES}

1. Ashby-Richardson H, Rogers GS, Stadecker MJ. Superficial acral fibromyxoma: an overview. Arch Pathol Lab Med. 2011;135:1064-6.

2. Wakabayashi $Y$, Nakai N, Takenaka H, Katoh N. Superficial acral fibromyxoma of the great toe: case report and mini-review of the literature. Acta Dermatovenerol Croat. 2012;20:263-6.

3. Messeguer F, Nagore E, Agustí-Mejias A, Traves V. Fibromixoma acral superficial, un tumor periungueal CD34 positivo. Actas Dermosifiliogr. 2012;103:67-9.

4. Fetsch JF, Laskin WB, Miettinen M. Superficial acral fibromyxoma: a clinicopathologic and immunohistochemical analysis of 37 cases of a distinctive soft tissue tumor with a predilection for the fingers and toes. Hum Pathol. 2001;32:704-14.

5. Wei C, Fleegler EJ. Superficial acral fibromyxoma of the thumb. Eplasty. 2013;13:ic13

6. Prescott RJ, Husain EA, Abdellaoui A, Al-Mahmoud RM, Khan M, Salman WD, et al. Superficial acral fibromyxoma: a clinicopathological study of 41 new cases from the U.K.: should myxoma (NOS) and fibroma (NOS) continue as part of 21stcentury reporting? Br J Dermatol. 2008;159:1315-21

7. Al-Daraji WI, Miettinen M.. Superficial acral fibromyxoma: a clinicopathological analysis of 32 tumors including 4 in the heel. J Cutan Pathol. 2008 Nov;35(11):1020-6.

How to cite this article: Márquez García A, Mendonça FMI, Perea Cejudo M, Camacho Martínez FM, Ríos Martín JJ. Superficial Acral Fibromyxoma involving the nail 's apparatus. Case report and literature review. An Bras Dermatol. 2014;89(1):147-9. 